\title{
The Laguerre pseudospectral method for the two-dimensional Schrödinger equation with symmetric nonseparable potentials
}

\author{
Haydar Alıci (i) \\ Department of Mathematics, Harran University, 63290, Sanluurfa, Turkey
}

\begin{abstract}
The Hermite pseudospectral method is one of the natural techniques for the numerical treatment of the problems defined over unbounded domains such as two-dimensional timeindependent Schrödinger equation on the whole real plane. However, it is shown here that for the symmetric potentials, transformation of the problem over the first quadrant and the application of the Laguerre pseudospectral method reduce the cost by a factor of four when compared to the Hermite pseudospectral method.
\end{abstract}

Mathematics Subject Classification (2010). 65L60, 81Q05, 65L15, 34L40, 42C10

Keywords. the Laguerre pseudospectral method, two dimensional Schrödinger equation, symmetric potentials

\section{Introduction}

In [1], the eigenvalues and wavefunctions of the two-dimensional time-independent Schrödinger equation

$$
\left[-\frac{\partial^{2}}{\partial x^{2}}-\frac{\partial^{2}}{\partial y^{2}}+V(x, y)\right] \Psi(x, y)=E \Psi(x, y), \quad(x, y) \in \mathbb{R}^{2}
$$

with the boundary conditions

$$
\begin{aligned}
& \Psi(x, \pm \infty)=0, \quad-\infty<x<\infty \\
& \Psi( \pm \infty, y)=0, \quad-\infty<y<\infty
\end{aligned}
$$

are approximated by means of the Hermite pseudospectral method (HPM) for several nonseparable quantum mechanical potentials $V(x, y)$. In this article, we consider the reflection symmetric potentials

$$
V(x, y)=V(-x, y)=V(x,-y)=V(-x,-y)
$$

for which the spectrum of the system (1.1)-(1.2) can be decomposed into four subsets containing the states $E_{2 n, 2 m}, E_{2 n, 2 m+1}, E_{2 n+1,2 m}$ and $E_{2 n+1,2 m+1}$. Although the Hermite pseudospectral method can still be used with further modifications in the Lagrange interpolant so that each eigenvalue subset can be treated by a separate basis set, it does not seem practical. Therefore, the main aim of this paper is to find more natural basis sets which can treat the states $E_{2 n, 2 m}, E_{2 n, 2 m+1}, E_{2 n+1,2 m}$ and $E_{2 n+1,2 m+1}$ separately. In this

Email address: haydara@harran.edu.tr

Received: 13.09.2018; Accepted: 14.01.2019 
case, one deals with four matrices of size $N^{2} \times N^{2}$ instead of a matrix of size $(2 N)^{2} \times(2 N)^{2}$ reducing the cost by a factor of two in each direction which is a considerable amount in two dimensions.

First of all the reflection symmetric character of the potential suggests the use of transformations

$$
\xi=(\alpha x)^{2}, \quad \eta=(\alpha y)^{2}, \quad \alpha>0
$$

where the parameter $\alpha$ may be regarded as an optimization parameter and introduced for numerical purposes. With (1.4), the Schrödinger equation takes the form

$$
\left[\xi \frac{\partial^{2}}{\partial \xi^{2}}+\eta \frac{\partial^{2}}{\partial \eta^{2}}+\frac{1}{2} \frac{\partial}{\partial \xi}+\frac{1}{2} \frac{\partial}{\partial \eta}-\frac{1}{4 \alpha^{2}} V(\sqrt{\xi} / \alpha, \sqrt{\eta} / \alpha)\right] \Psi=-\frac{E}{4 \alpha^{2}} \Psi
$$

where $(\xi, \eta) \in(0, \infty) \times(0, \infty)$. Accordingly the boundary conditions in (1.2) read as

$$
\begin{array}{ll}
\Psi(\xi, \infty)=0, & 0<\xi<\infty \\
\Psi(\infty, \eta)=0, & 0<\eta<\infty .
\end{array}
$$

Now, introducing the transformation

$$
\Psi(\xi, \eta)=\xi^{s_{1}} \eta^{s_{2}} e^{-\frac{1}{2}(\xi+\eta)} \Phi(\xi, \eta), \quad s_{1}, s_{2} \geq 0
$$

satisfying the boundary conditions in (6) where the polynomial terms are introduced to cope with the artificial singularity at $\xi=0$ and $\eta=0$, we rewrite the last equation as

$$
\left[\xi \frac{\partial^{2}}{\partial \xi^{2}}+\eta \frac{\partial^{2}}{\partial \eta^{2}}+\left(2 s_{1}+\frac{1}{2}-\xi\right) \frac{\partial}{\partial \xi}+\left(2 s_{2}+\frac{1}{2}-\eta\right) \frac{\partial}{\partial \eta}+Q\right] \Phi=\varepsilon \Phi
$$

where

$$
Q=\frac{s_{1}\left(s_{1}-1 / 2\right)}{\xi}+\frac{s_{2}\left(s_{2}-1 / 2\right)}{\eta}+\frac{\xi+\eta}{4}-\frac{1}{4 \alpha^{2}} V(\sqrt{\xi} / \alpha, \sqrt{\eta} / \alpha)
$$

stands for the modified potential and

$$
\mathcal{E}=\mathcal{E}\left(s_{1}, s_{2}, \alpha\right)=s_{1}+s_{2}+\frac{1}{2}-\frac{E}{4 \alpha^{2}}
$$

the shifted eigenvalues. Notice from (1.7) that the new dependent variable $\Phi$ does not have to satisfy any boundary condition as long as it is a bounded function of $\xi$ and $\eta$.

Then, to get rid of the unwelcome terms that are proportional to $\xi^{-1}$ and $\eta^{-1}$ appearing in the modified potential, we are free to choose any one of the elements from the set $\left\{(0,0),\left(0, \frac{1}{2}\right),\left(\frac{1}{2}, 0\right),\left(\frac{1}{2}, \frac{1}{2}\right)\right\}$ for $\left(s_{1}, s_{2}\right)$. Moreover, setting

$$
2 s_{i}+\frac{1}{2}=\gamma_{i}+1, \quad i=1,2
$$

the last equation reads as

$$
\left[\xi \frac{\partial^{2}}{\partial \xi^{2}}+\eta \frac{\partial^{2}}{\partial \eta^{2}}+\left(\gamma_{1}+1-\xi\right) \frac{\partial}{\partial \xi}+\left(\gamma_{2}+1-\eta\right) \frac{\partial}{\partial \eta}+Q\right] \Phi=\varepsilon \Phi
$$

where the modified potential

$$
Q=Q(\xi, \eta ; \alpha)=\frac{1}{4}\left[\xi+\eta-\frac{1}{\alpha^{2}} V(\sqrt{\xi} / \alpha, \sqrt{\eta} / \alpha)\right]
$$

is now free of the parameters $\gamma_{i}$, and hence $s_{i}$, for $i=1,2$. Meanwhile, the shifted eigenvalues becomes

$$
\mathcal{E}=\mathcal{E}\left(\gamma_{1}, \gamma_{2}, \alpha\right)=\frac{1}{2}\left(\gamma_{1}+\gamma_{2}\right)+1-\frac{E}{4 \alpha^{2}} .
$$

Notice from (1.7) that the $\left(\gamma_{1}, \gamma_{2}\right) \in\left\{\left(-\frac{1}{2},-\frac{1}{2}\right),\left(-\frac{1}{2}, \frac{1}{2}\right),\left(\frac{1}{2},-\frac{1}{2}\right),\left(\frac{1}{2}, \frac{1}{2}\right)\right\}$ values obtained from (1.11) lead to the states $E_{2 m, 2 n}, E_{2 m, 2 n+1}, E_{2 m+1,2 n}$ and $E_{2 m+1,2 n+1}$ respectively. This can be seen on returning back to the original variables $(x, y)$ via (1.4). 
Note that the Schrödinger equation in (1.1) is defined over the whole real plane whereas the transformed equation (1.12) of a reflection symmetric system is defined over the first quadrant. Thus, the direct application of the HPM used in [1] is not possible anymore. Instead, Laguerre pseudospectral method (LPM) seems more feasible since the Laguerre polynomials constitute a complete orthogonal set on the half line $(0, \infty)$.

Therefore, in section 2 we construct the pseudospectral formulation of the transformed equation in (1.12) based on the Laguerre polynomials. Section 3 includes numerical examples and some implementation notes. The last section concludes the paper with some remarks.

\section{Pseudospectral formulation of the problem}

Since a pseudospectral method is based on a polynomial interpolation in the Lagrange form, we propose an approximate solution of the form

$$
\Phi(\xi, \eta)=\sum_{n=0}^{N} \sum_{j=0}^{N} \ell_{n}(\xi) \ell_{j}(\eta) \Phi_{n j}
$$

where

$$
\ell_{k}(\xi)=\frac{\phi_{N+1}(\xi)}{\left(\xi-\xi_{k}\right) \phi_{N+1}^{\prime}\left(\xi_{k}\right)}, \quad k=0,1, \ldots, N
$$

are the set of $N$-th degree Lagrange interpolating polynomials in which

$$
\phi_{N+1}(\xi)=k_{N+1} \prod_{i=0}^{N}\left(\xi-\xi_{i}\right), \quad k_{N+1} \in \mathbb{R}
$$

is the $(N+1)$-st degree polynomial having real and distinct zeros. Here, $\Phi_{n j}=\Phi\left(\xi_{n}, \eta_{j}\right)$ are the exact values of $\Phi(\xi, \eta)$ at the specified grid points $\left(\xi_{n}, \eta_{j}\right)$ for $n, j=0,1, \ldots, N$. Now to approximate the eigenvalues of the Schrödinger equation with symmetric potentials we set

$$
\phi_{N+1}^{\gamma}(t)=\frac{L_{N+1}^{\gamma}(t)}{h_{N+1}}, \quad h_{k}^{2}=\frac{\Gamma(k+\gamma+1)}{k !}, \quad t \in(0, \infty)
$$

in which $L_{N+1}^{\gamma}(t)$ is the Laguerre polynomial of degree $N+1$ and order $\gamma= \pm \frac{1}{2}$. Approximating the solutions of differential equations by Laguerre polynomials are usually not stable for large $N$ due to their wild behaviors at infinity, and hence, one usually works with the Laguerre functions $\hat{L}_{N}^{\gamma}(t)=e^{-t / 2} L_{N}^{\gamma}(t)$ instead. This situation is theoretically investigated, for example in [5,7-9], and it is shown that the Laguerre functions have better stability properties than Laguerre polynomials. In this study, we search for the square integrable solutions of (1.1) which vanish exponentially at infinity. Therefore, the Laguerre functions are suitable for the numerical treatment of (1.1). But, since we factor off the term $e^{-(\xi+\eta) / 2}$ from the solution by means of the transformation in (1.7), the use of Laguerre polynomials for equation (1.12) is equivalent to the use of Laguerre functions for the original problem in (1.1). Thus, we continue with the normalized Laguerre polynomials in (2.4) to approximate the eigenvalues of (1.12) and hence, those of the original equation in (1.1).

By (2.4) the grid points $\left(\xi_{n}, \eta_{j}\right)$ are set to be the cartesian product of the zeros $\xi_{n}$ and $\eta_{j}$ of the Laguerre polynomials $L_{N+1}^{\gamma_{1}}(\xi)$ and $L_{N+1}^{\gamma_{2}}(\eta)$, respectively. That is, we have four set of grid points according to the choice of the parameters

$$
\left(\gamma_{1}, \gamma_{2}\right) \in\left\{\left(-\frac{1}{2},-\frac{1}{2}\right),\left(-\frac{1}{2}, \frac{1}{2}\right),\left(\frac{1}{2},-\frac{1}{2}\right),\left(\frac{1}{2}, \frac{1}{2}\right)\right\}
$$

which will be used to approximate the states $E_{2 m, 2 n}, E_{2 m, 2 n+1}, E_{2 m+1,2 n}$ and $E_{2 m+1,2 n+1}$ respectively. 
On the other hand, zeros of the normalized Laguerre polynomials may be determined to a desired accuracy as the eigenvalues of the tridiagonal matrix

$$
\mathbf{R}=\left[\begin{array}{ccccc}
B_{0} & A_{1} & & & 0 \\
A_{1} & B_{1} & A_{2} & & \\
& A_{2} & B_{2} & \ddots & \\
& & \ddots & \ddots & A_{N} \\
0 & & & A_{N} & B_{N}
\end{array}\right]
$$

where the $A_{n}=-\sqrt{n(n+\gamma)}$ and $B_{n}=2 n+\gamma+1$ are the coefficients in the three term recursion

$$
A_{n+1} \phi_{n+1}^{\gamma}(\xi)+\left(B_{n}-\xi\right) \phi_{n}^{\gamma}(\xi)+A_{n} \phi_{n-1}^{\gamma}(\xi)=0, \quad n=0,1, \ldots
$$

of the normalized Laguerre polynomials [2,12]. Actually, the matrix $\mathbf{R}$ is obtained by running the recursion from $n=0$ to $n=N$ and setting $\phi_{N+1}(\xi)$ to zero. Thus, the $m$ th computed eigenvector $\mathbf{r}_{m}=\left[\begin{array}{lllll}r_{0, m} & r_{1, m} & \ldots & r_{N-1, m} & r_{N, m}\end{array}\right]^{T}$ of $\mathbf{R}$ corresponding to the $m$-th eigenvalue (or root) $\xi_{m}$ is a constant multiple of the vector of orthonormal polynomial values $\phi^{\gamma m}=\left[\phi_{0}^{\gamma}\left(\xi_{m}\right) \phi_{1}^{\gamma}\left(\xi_{m}\right) \ldots \phi_{N-1}^{\gamma}\left(\xi_{m}\right) \phi_{N}^{\gamma}\left(\xi_{m}\right)\right]^{T}$ at the point $\xi_{m}$. That is, $\mathbf{r}_{m}=a \phi^{m}$. This constant may be determined by comparing the first elements $r_{0, m}$ and $\phi_{0}^{\gamma}\left(\xi_{m}\right)$ of these two vectors since $\phi_{0}^{\gamma}\left(\xi_{m}\right)=1 / h_{0}=1 / \sqrt{\Gamma(\gamma+1)}$ is a constant. Therefore, for $n=0,1, \ldots, N$, the values $\phi_{n}^{\gamma}\left(\xi_{m}\right)$ of the normalized classical orthogonal polynomials at the zeros of $\phi_{N+1}^{\gamma}(\xi)$ may be computed as

$$
\begin{aligned}
{\left[\phi_{0}^{\gamma}\left(\xi_{m}\right) \phi_{1}^{\gamma}\left(\xi_{m}\right) \ldots \phi_{N-1}^{\gamma}\left(\xi_{m}\right) \phi_{N}^{\gamma}\left(\xi_{m}\right)\right]^{T}=} \\
\frac{1}{\sqrt{\Gamma(\gamma+1)} r_{0, m}}\left[r_{0, m} r_{1, m} \ldots r_{N-1, m} r_{N, m}\right]^{T}
\end{aligned}
$$

in terms of the computed eigenvector $\mathbf{r}_{m}$ of tridiagonal symmetric matrix $\mathbf{R}$ of size $N+1$.

It is known as the Golub-Welsch algorithm whose details may be found, for example, in $[2,4]$. This procedure, unfortunately, suffers from computing the full set of eigenvalues with a uniform accuracy especially for large values of $N$. Therefore, in this case, alternative root finding algorithms, for example, Newton method may be used to compute the roots. Nevertheless, for moderate $N$ values the Golub-Welsch algorithm can be used without any hesitation.

After determining the mesh points $\left(\xi_{n}, \eta_{j}\right)$, we insert the approximate solution in (2.1) into the equation (1.12) and require its satisfaction at the grid points $\left(\xi_{m}, \eta_{i}\right)$ to obtain the set of $(N+1)^{2}$ equations

$$
\begin{aligned}
\sum_{n=0}^{N} \sum_{j=0}^{N}\left[\widehat{L}_{n}\left(\xi_{m}\right) \ell_{j}\left(\eta_{i}\right)+\widehat{L}_{j}\left(\eta_{i}\right) \ell_{n}\left(\xi_{m}\right)+Q\left(\xi_{m}, \eta_{i} ; \alpha\right) \ell_{n}\left(\xi_{m}\right) \ell_{j}\left(\eta_{i}\right)\right] \Phi_{n j} \\
=\mathcal{E}\left(\gamma_{1}, \gamma_{2}, \alpha\right) \sum_{n=0}^{N} \sum_{j=0}^{N} \ell_{n}\left(\xi_{m}\right) \ell_{j}\left(\eta_{i}\right) \Phi_{n j}
\end{aligned}
$$

for $m, i=0,1, \ldots, N$ where

$$
\widehat{L}_{r}\left(\zeta_{p}\right):=\widehat{L}_{p r}^{\gamma}=\zeta_{p} \ell_{r}^{\prime \prime}\left(\zeta_{p}\right)+\left(\gamma+1-\zeta_{p}\right) \ell_{r}^{\prime}\left(\zeta_{p}\right) .
$$

Here, notice the dependence of the roots to the order $\gamma$ of the Laguerre polynomials. That is, the points $\zeta_{p}:=\zeta_{p}^{\gamma}$ are the roots of the $(N+1)$ st degree Laguerre polynomials of order $\gamma$. 
Therefore, by using (2.9) and the well-known property $\ell_{r}\left(\zeta_{p}\right)=\delta_{p r}$ of the Lagrange polynomials, the algebraic equations in (2.8) reads as

$$
\begin{aligned}
\sum_{n=0}^{N} \sum_{j=0}^{N}\left(\widehat{L}_{m n}^{\gamma_{1}} \delta_{i j}+\widehat{L}_{i j}^{\gamma_{2}} \delta_{m n}+Q_{m i} \delta_{m n} \delta_{i j}\right) \Phi_{n j}= & \\
& \mathcal{E}\left(\gamma_{1}, \gamma_{2}, \alpha\right) \sum_{n=0}^{N} \sum_{j=0}^{N} \delta_{m n} \delta_{i j} \Phi_{n j}
\end{aligned}
$$

where the $Q_{m i}=Q\left(\xi_{m}, \eta_{i} ; \alpha\right)$ are the known values of the modified potential in (1.13) at the mesh points. The last equation may be written in the matrix-vector form

$$
\widehat{\mathbf{B}} \boldsymbol{\Phi}=\mathcal{E}\left(\gamma_{1}, \gamma_{2}, \alpha\right) \boldsymbol{\Phi}
$$

where

$$
\widehat{\mathbf{B}}\left(\gamma_{1}, \gamma_{2}\right):=\widehat{\mathbf{B}}=\widehat{\mathbf{L}}^{\gamma_{\mathbf{1}}} \otimes \mathbf{I}+\mathbf{I} \otimes \widehat{\mathbf{L}}^{\gamma_{\mathbf{2}}}+\mathbf{Q}_{\mathbf{1}}
$$

is a matrix of dimension $(N+1)^{2} \times(N+1)^{2}$. Here $\boldsymbol{\Phi}$ is an $(N+1)^{2} \times 1$ vector containing the vectorized unknown wavefunction values at the grid points in the order

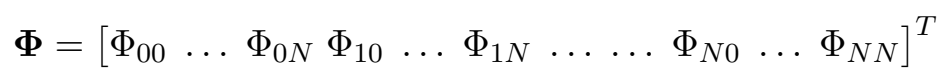

where $\Phi_{i j}$ stand for the values $\Phi\left(\xi_{i}, \eta_{j}\right)$ of the wavefunction at the nodal points $\left(\xi_{i}, \eta_{j}\right)$. Moreover, $\widehat{\mathbf{L}}^{\gamma_{\mathbf{i}}}$ for $i=1,2$ stands for the kinetic energy matrix with entries (2.9), I the $(N+1) \times(N+1)$ identity matrix, $\mathbf{Q}$ the diagonal potential matrix whose diagonal entries contain the values $Q\left(\xi_{m}, \eta_{i} ; \alpha\right)$ in the order specified in (2.13) and $\mathbf{M}_{1} \otimes \mathbf{M}_{2}$ the Kronecker product of the matrices $\mathbf{M}_{1}$ and $\mathbf{M}_{2}$.

The explicit entries [12]

$$
\widehat{L}_{p r}^{\gamma}=-\frac{1}{6} \begin{cases}\frac{12 \zeta_{p}}{\left(\zeta_{p}-\zeta_{r}\right)^{2}} \frac{\phi_{N+1}^{\gamma \prime}\left(\zeta_{p}\right)}{\phi_{N+1}^{\gamma}\left(\zeta_{r}\right)} & \text { if } p \neq r \\ 2 N+\frac{1}{\zeta_{r}}\left[\left(\gamma-\zeta_{r}\right)^{2}-1\right] & \text { if } p=r\end{cases}
$$

of the matrix $\widehat{\mathbf{L}}^{\gamma}$ in (2.9) reveal that it is, and hence $\widehat{\mathbf{B}}$, is not symmetric. However, fortunately, the similarity transformation

$$
\mathbf{L}^{\gamma}=\left(\mathbf{S}^{\gamma}\right)^{-1} \widehat{\mathbf{L}}^{\gamma} \mathbf{S}^{\gamma}
$$

in which

$$
\mathbf{S}^{\gamma}=s_{p} \delta_{p r}=\sqrt{\zeta_{p}} \phi_{N+1}^{\gamma}{ }^{\prime}\left(\zeta_{p}\right) \delta_{p r}
$$

is a diagonal matrix symmetrizes the matrix $\widehat{\mathbf{L}}^{\gamma}$. In this case, the entries of the symmetric matrix $\mathbf{L}^{\gamma}$ reads as

$$
L_{p r}^{\gamma}=-\frac{1}{6} \begin{cases}\frac{12 \sqrt{\zeta_{p} \zeta_{r}}}{\left(\zeta_{p}-\zeta_{r}\right)^{2}} & \text { if } p \neq r \\ 2 N+\frac{1}{\zeta_{r}}\left[\left(\gamma-\zeta_{r}\right)^{2}-1\right] & \text { if } p=r\end{cases}
$$

with $K_{p r}^{\gamma}=K_{r p}^{\gamma}$. Therefore, we may state the following proposition.

Proposition 2.1. The matrix

$$
\mathbf{T}=\mathbf{S}^{\gamma_{1}} \otimes \mathbf{S}^{\gamma_{2}}
$$

in which $\mathbf{S}^{\gamma}$ is given by (2.16), symmetrizes the matrix $\widehat{\mathbf{B}}$ in (2.12). 
Proof. By using the basic linear algebra tools

$$
(\mathbf{A} \otimes \mathbf{B})^{-1}=\mathbf{A}^{-1} \otimes \mathbf{B}^{-1} \quad \text { and } \quad(\mathbf{A} \otimes \mathbf{B})(\mathbf{C} \otimes \mathbf{D})=\mathbf{A} \mathbf{C} \otimes \mathbf{B D}
$$

and after a little algebra we end up with

$$
\begin{aligned}
\mathbf{B}= & \mathbf{T}^{-1} \widehat{\mathbf{B}} \mathbf{T}=\left(\mathbf{S}^{\gamma_{1}} \otimes \mathbf{S}^{\gamma_{2}}\right)^{-1}\left(\widehat{\mathbf{L}}^{\gamma_{1}} \otimes \mathbf{I}+\mathbf{I} \otimes \widehat{\mathbf{L}}^{\gamma_{2}}+\mathbf{Q}\right)\left(\mathbf{S}^{\gamma_{1}} \otimes \mathbf{S}^{\gamma_{2}}\right) \\
= & \left(\mathbf{S}^{\gamma_{1}}\right)^{-1} \widehat{\mathbf{L}}^{\gamma_{1}} \mathbf{S}^{\gamma_{1}} \otimes \mathbf{I}+\mathbf{I} \otimes\left(\mathbf{S}^{\gamma_{2}}\right)^{-1} \widehat{\mathbf{L}}^{\gamma_{2}} \mathbf{S}^{\gamma_{2}} \\
& +\left(\mathbf{S}^{\gamma_{1}} \otimes \mathbf{S}^{\gamma_{2}}\right)^{-\mathbf{1}} \mathbf{Q}\left(\mathbf{S}^{\gamma_{1}} \otimes \mathbf{S}^{\gamma_{2}}\right) .
\end{aligned}
$$

Now using (2.15) for the first two term and keeping in mind that $\mathbf{T}=\mathbf{S}^{\gamma_{\mathbf{1}}} \otimes \mathbf{S}^{\gamma_{\mathbf{2}}}$ and $\mathbf{Q}$ are diagonal matrices the last equation reads as

$$
\mathbf{B}=\mathbf{L}^{\gamma_{1}} \otimes \mathbf{I}+\mathbf{I} \otimes \mathbf{L}^{\gamma_{2}}+\mathbf{Q} .
$$

Hence, being the addition of symmetric matrices, $\mathbf{B}$ is symmetric.

Thus we may replace the unsymmetrical system in (2.11) with the simple symmetric one

$$
\mathbf{B u}=\mathcal{E} \mathbf{u}
$$

since the similar matrices participate of the same eigenvalue set. It is simple in the sense that the unpleasant term $\phi_{N+1}^{\prime}\left(\zeta_{p}\right) / \phi_{N+1}^{\prime}\left(\zeta_{r}\right)$ in $(2.14)$ is removed by the similarity transformation in (2.18). Notice that the eigenvectors of the unsymmetrical and symmetric systems are linked as

$$
\Phi=\mathbf{T u}
$$

since $\mathbf{B u}=\mathbf{T}^{-1} \widehat{\mathbf{B}} \mathbf{T u}=\mathcal{E} \mathbf{u}$ implies $\widehat{\mathbf{B}}[\mathbf{T u}]=\mathcal{E}[\mathbf{T u}]$. The last equation may be written in nodal form as

$$
\Phi\left(\xi_{m}, \eta_{i}\right)=\sqrt{\xi_{m} \eta_{i}} \phi_{N+1}^{\gamma_{1}{ }^{\prime}}\left(\xi_{m}\right) \phi_{N+1}^{\gamma_{2}{ }^{\prime}}\left(\eta_{i}\right) u_{m i}
$$

with the help of (2.16) and (2.18). Then, (1.7) together with (1.11) and (2.23) leads to the values of the original wavefunction

$$
\Psi\left(\xi_{m}, \eta_{i}\right)=\phi_{N+1}^{\gamma_{1}}\left(\xi_{m}\right) \phi_{N+1}^{\gamma_{2}{ }^{\prime}}\left(\eta_{i}\right) \xi_{m}^{\frac{1}{2}\left(\gamma_{1}+\frac{3}{2}\right)} \eta_{i}^{\frac{1}{2}\left(\gamma_{2}+\frac{3}{2}\right)} e^{-\frac{1}{2}(\xi+\eta)} u_{m i}
$$

at the mesh point $\left(\xi_{m}, \eta_{i}\right)$ in terms of an eigenvector $\mathbf{u}$ of (2.21). On the other hand, setting $n=N+1$ and $x=\xi_{m}, \eta_{i}$ in the differential difference relation

$$
x \phi_{n}^{\gamma{ }^{\prime}}(x)=n \phi_{n}^{\gamma}{ }^{\prime}(x)-\sqrt{n(n+\gamma)} \phi_{n-1}^{\gamma \prime}(x)
$$

of the normalized Laguerre polynomials we obtain

$$
\begin{aligned}
\xi_{m} \phi_{N+1}^{\gamma_{1}{ }^{\prime}}\left(\xi_{m}\right) & =-\sqrt{(N+1)\left(N+\gamma_{1}+1\right)} \phi_{N}^{\gamma_{1}}\left(\xi_{m}\right) \\
\eta_{i} \phi_{N+1}^{\gamma_{2}}{ }^{\prime}\left(\eta_{i}\right) & =-\sqrt{(N+1)\left(N+\gamma_{2}+1\right)} \phi_{N}^{\gamma_{2}}\left(\eta_{i}\right)
\end{aligned}
$$

since $\phi_{N+1}^{\gamma_{1}}\left(\xi_{m}\right)=\phi_{N+1}^{\gamma_{2}}\left(\eta_{i}\right)=0$. Now, using (2.7) and (2.26), equation (2.24) reads as

$$
\Psi\left(\xi_{m}, \eta_{i}\right)=C\left(N, \gamma_{1}, \gamma_{2}\right) \frac{r_{N, m}}{r_{0, m}} \frac{r_{N, i}}{r_{0, i}} \xi_{m}^{\frac{1}{2}\left(\gamma_{1}-\frac{1}{2}\right)} \eta_{i}^{\frac{1}{2}\left(\gamma_{2}-\frac{1}{2}\right)} e^{-\frac{1}{2}\left(\xi_{m}+\eta_{i}\right)} u_{m i}
$$

where

$$
C\left(N, \gamma_{1}, \gamma_{2}\right)=(N+1) \sqrt{\frac{\left(N+\gamma_{1}+1\right)\left(N+\gamma_{2}+1\right)}{\Gamma\left(\gamma_{1}+1\right) \Gamma\left(\gamma_{2}+1\right)}} .
$$

Now, we are in a position to state the following theorem: 
Theorem 2.2. The approximate eigenvalues $E_{k}$ of the two-dimensional Schrödinger equation in (1.1) together with the boundary condition (1.2) are related with the eigenvalues $\mathcal{E}_{k}$ of the matrix $\mathbf{B}\left(\gamma_{1}, \gamma_{2}\right)$ in (2.21) by the formula

$$
E_{k}=4 \alpha^{2}\left[\frac{1}{2}\left(\gamma_{1}+\gamma_{2}\right)+1-\varepsilon_{k}\left(\gamma_{1}, \gamma_{2}, \alpha\right)\right], \quad k=0,1, \ldots
$$

and the values of the corresponding normalized eigenfunctions $\bar{\Psi}_{k}\left(x_{n}, y_{j}\right)$ (in $L^{2}$ sense) at the mesh points in the first quadrant $\left(x_{n}, y_{j}\right)=\left(\sqrt{\xi_{n}} / \alpha, \sqrt{\eta_{j}} / \alpha\right)$ are given by

$$
\bar{\Psi}_{k}\left(x_{m}, y_{i}\right)=\alpha C\left(N, \gamma_{1}, \gamma_{2}\right) \frac{r_{N, m}}{r_{0, m}} \frac{r_{N, i}}{r_{0, i}} \xi_{m}^{\frac{1}{2}\left(\gamma_{1}-\frac{1}{2}\right)} \eta_{i}^{\frac{1}{2}\left(\gamma_{2}-\frac{1}{2}\right)} e^{-\frac{1}{2}\left(\xi_{m}+\eta_{i}\right)} u_{m i}^{k}
$$

provided that $\boldsymbol{u}^{k}$ is the $k$-th normalized (in Euclidean 2-norm) eigenvector of (2.21). Finally, the complete picture of the normalized eigenfunction $\bar{\Psi}_{k}(x, y)$ in the whole plane, at the original mesh points $\left(x_{m}, y_{i}\right)=\left( \pm \sqrt{\xi_{n}} / \alpha, \pm \sqrt{\eta_{j}} / \alpha\right)$ is obtained by appropriately (even and/or odd) extending the values in (2.30) to the other quadrants.

Proof. The first part easily follows from (1.10) and (1.11). For the second part, keeping in mind that for symmetric potentials the squares of the eigenfunctions are equal in all quadrants and then using the transformations in (1.4) and (1.7) we write

$$
\begin{aligned}
\left\|\bar{\Psi}_{k}\right\|_{L^{2}}^{2} & =\iint_{\mathbb{R}^{2}} \bar{\Psi}_{k}^{2}(x, y) \mathrm{d} x \mathrm{~d} y=4 \iint_{\mathbb{R}^{+2}} \bar{\Psi}_{k}^{2}(x, y) \mathrm{d} x \mathrm{~d} y \\
& =4 \alpha^{2} \iint_{\mathbb{R}^{+2}} \Psi_{k}^{2}(x, y) \mathrm{d} x \mathrm{~d} y=4 \alpha^{2} \iint_{\mathbb{R}^{+2}} \Psi_{k}^{2}(\xi, \eta) \frac{1}{4 \alpha^{2} \sqrt{\xi \eta}} \mathrm{d} \xi \mathrm{d} \eta \\
& =\iint_{\mathbb{R}^{+2}} \Phi_{k}^{2}(\xi, \eta) \xi^{\gamma_{1}} \eta^{\gamma_{2}} e^{-(\xi+\eta)} \mathrm{d} \xi \mathrm{d} \eta
\end{aligned}
$$

Now, applying the $\mathrm{N}+1$ point Gauss-Laguerre quadrature to the last integral we have

$$
\left\|\bar{\Psi}_{k}\right\|_{L^{2}}^{2}=\lim _{N \rightarrow \infty} \sum_{m=0}^{N} \sum_{i=0}^{N} \Phi_{k}^{2}\left(\xi_{m}, \xi_{i}\right) \omega_{m}^{\gamma_{1}} \omega_{i}^{\gamma_{2}}
$$

where

$$
\begin{aligned}
& \omega_{m}^{\gamma_{1}}=\frac{1}{(N+1)\left(N+\gamma_{1}+1\right)} \frac{\xi_{m}}{\phi_{N}^{\gamma_{1}}\left(\xi_{m}\right)}, \quad m=0,1, \ldots, N \\
& \omega_{i}^{\gamma_{2}}=\frac{1}{(N+1)\left(N+\gamma_{2}+1\right)} \frac{\eta_{i}}{\phi_{N}^{\gamma_{2}^{2}}\left(\eta_{i}\right)}, \quad i=0,1, \ldots, N
\end{aligned}
$$

are known as the Christofell numbers of the Laguerre-Gauss quadrature in terms of the normalized Laguerre polynomials [6]. Now, plugging (2.26) into (2.23) we obtain

$$
\Phi\left(\xi_{m}, \eta_{i}\right)=(N+1) \sqrt{\left(N+\gamma_{1}+1\right)\left(N+\gamma_{2}+1\right)} \frac{\phi_{N}^{\gamma_{1}}\left(\xi_{m}\right) \phi_{N}^{\gamma_{2}}\left(\eta_{i}\right)}{\sqrt{\xi_{m} \eta_{i}}} .
$$

Finally, with the help of (2.34) and (2.33), (2.32) takes the form

$$
\left\|\bar{\Psi}_{k}\right\|_{L^{2}}^{2}=\lim _{N \rightarrow \infty} \sum_{n=0}^{N} \sum_{j=0}^{N}\left(u_{n j}^{k}\right)^{2}
$$

in which the term with double sum is the squared Euclidean 2-norm of the vector $\mathbf{u}_{(N+1)^{2} \times 1}^{k}$. Thus we have

$$
\left\|\bar{\Psi}_{k}\right\|_{L^{2}}^{2}=\lim _{N \rightarrow \infty}\left\|\mathbf{u}^{k}\right\|_{2}^{2}=\lim _{N \rightarrow \infty} 1=1
$$

since $\left\|\mathbf{u}^{k}\right\|_{2}=1$ by hypothesis which completes the proof. 


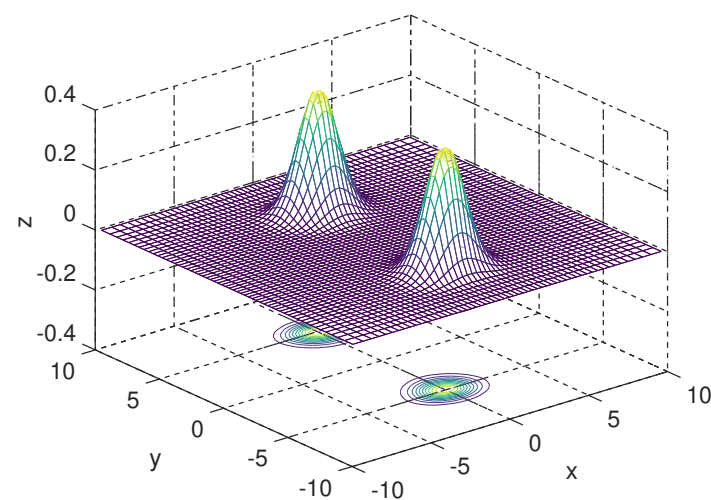

(a) $\bar{\Psi}_{00}(x, y), \quad E_{00}=2.2629597964443$

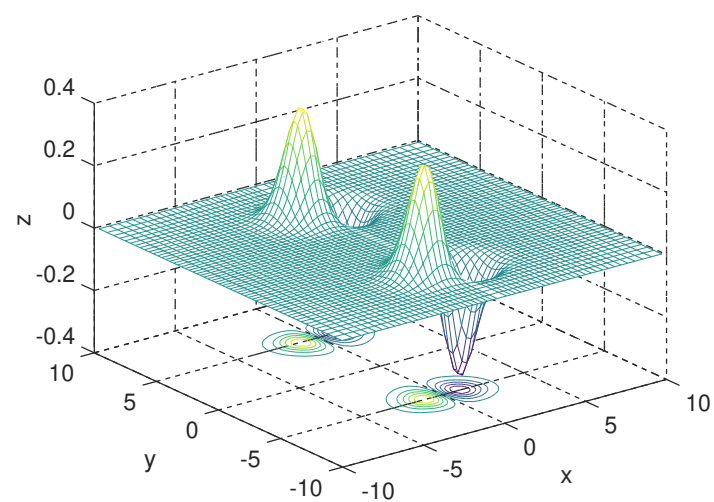

(c) $\bar{\Psi}_{01}(x, y), \quad E_{01}=3.9992895301372$

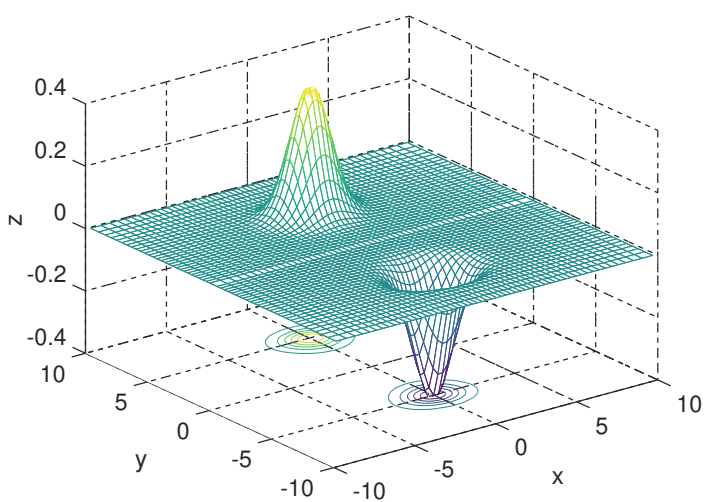

(b) $\bar{\Psi}_{10}(x, y), \quad E_{10}=2.2629597981908$

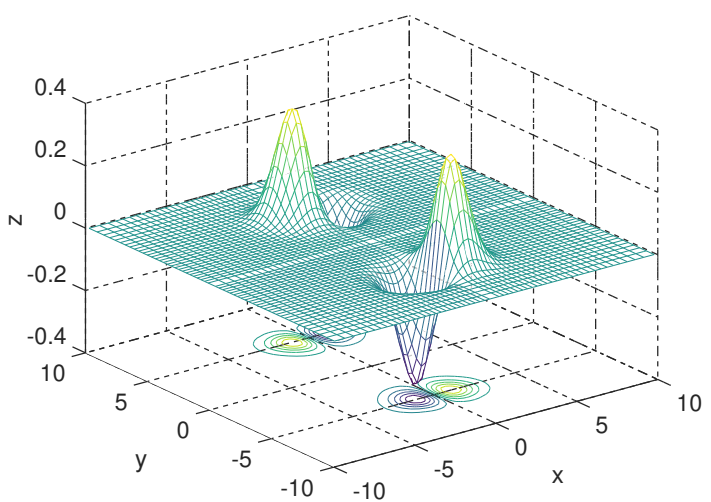

(d) $\bar{\Psi}_{11}(x, y), \quad E_{11}=3.9992895313331$

Figure 1. First four normalized wavefunctions of the symmetric double-well potential in (3.1) when $\mu=0.01$.

\section{Numerical results and discussion}

In this section, we apply the methods described in the previous section to several symmetric two dimensional quantum mechanical potentials. As a first example we consider the symmetric double-well potential

$$
V(x, y)=x^{2}-y^{2}+\mu y^{2}\left(2 y^{2}-x^{2}\right)+\frac{1}{8 \mu}
$$

where $\mu$ is a positive real parameter. In all tables, $n$ stands for the eigenvalue index, $N$ the truncation order for which the desired accuracy of the corresponding eigenvalue is obtained, and $\alpha_{\text {opt }}$ denotes the optimum value of the scaling or an optimization parameter $\alpha$ for which the desired accuracy is obtained with the smallest possible truncation order $N$. A method to determine the optimum value of $\alpha$ for Gaussian type functions is given in [10] which can also be applied in our problem since the eigenfunctions of (1.1) are of Gaussian type.

The accuracy of the results in all tables reported here has been checked by inspecting the number of stable digits between two consecutive truncation orders. Therefore, only the last digits might be incorrect because of rounding.

Table 1 presents the accuracy improvement of the ground state eigenvalue of the symmetric double well potential by systematically increasing the truncation size $N$. It is clear from Table 1 that the increase in $N$ by one results in an accuracy gain of one digit for 
Table 1. Accuracy improvement of the ground state eigenvalue of the symmetric double well potential in (3.1).

\begin{tabular}{|c|c|c|c|}
\hline$\mu$ & $\alpha_{o p t}$ & $N$ & $E_{0,0}$ \\
\hline \multirow[t]{7}{*}{0.01} & 1 & 12 & 2.261 \\
\hline & & 15 & 2.262956 \\
\hline & & 18 & 2.262959790 \\
\hline & & 21 & 2.26295979646 \\
\hline & & 24 & 2.26295979644469 \\
\hline & & 27 & 2.262959796444740 \\
\hline & & 28 & 2.262959796444740 \\
\hline
\end{tabular}

this potential. This is typical for all potentials considered here. In fact, for some specific potentials the accuracy gain is more than one digit when the truncation size is increased by one.

Table 2. Several nearly degenerate eigenvalues of the symmetric double-well potential in (3.1). For comparison, we include the corresponding results from [1] which uses the HPM. For both methods $\alpha_{o p t}=1$.

\begin{tabular}{|c|c|c|c|c|}
\hline$\mu$ & $N_{L P M}$ & $N_{H P M}$ & $E_{2 m, 2 n} L P M / H P M$ & $E_{2 m, 2 n+1} L P M / H P M$ \\
\hline$\overline{0.01}$ & 28 & 56 & 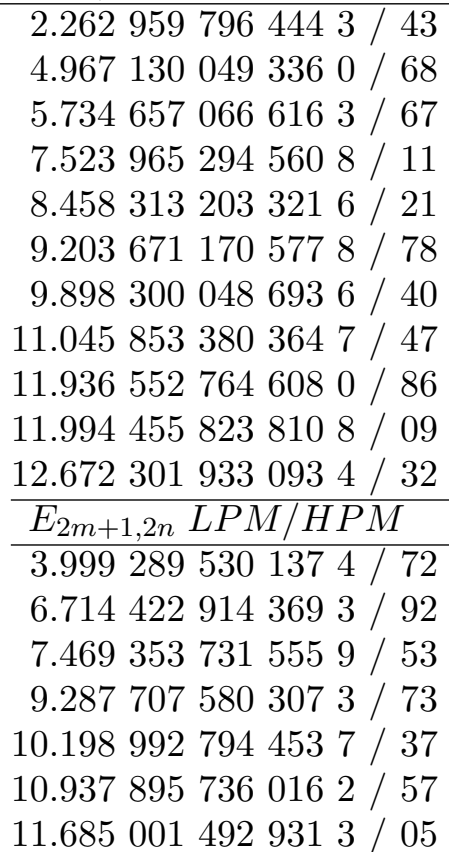 & 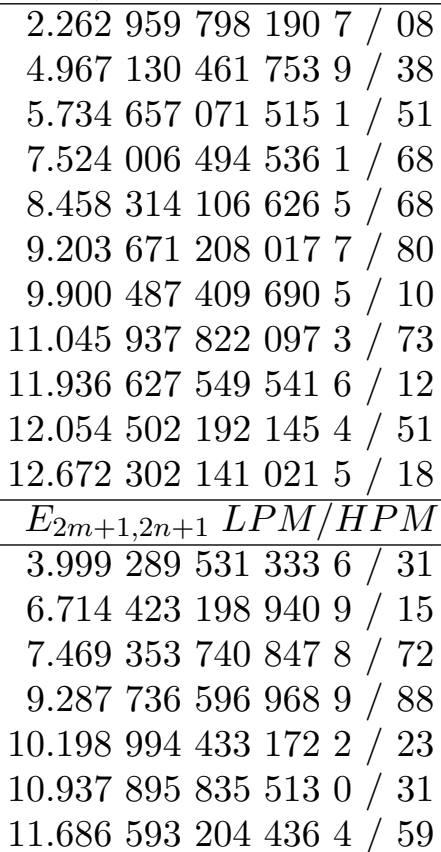 \\
\hline
\end{tabular}

Table 2 presents some nearly degenerate states of the symmetric double-well potential. For comparison we include the results of [1], which uses Hermite pseudospectral methods in both direction. It is clear from Table 2 that the use of Laguerre instead of Hermite polynomials halved the truncation size in each direction that is necessary to obtain the accuracy quoted. More clearly, by using the HPM we need to diagonalize a matrix of size $(2 N)^{2} \times(2 N)^{2}$ while with the use of LPM we only need to diagonalize four separate $N^{2} \times N^{2}$ matrices to get the same number of eigenvalues to the same accuracy.

As a second example, we take into account the Gaussian type potential

$$
V(x, y)=-e^{-\beta\left(x^{4}+y^{4}\right)}, \quad \beta>0
$$




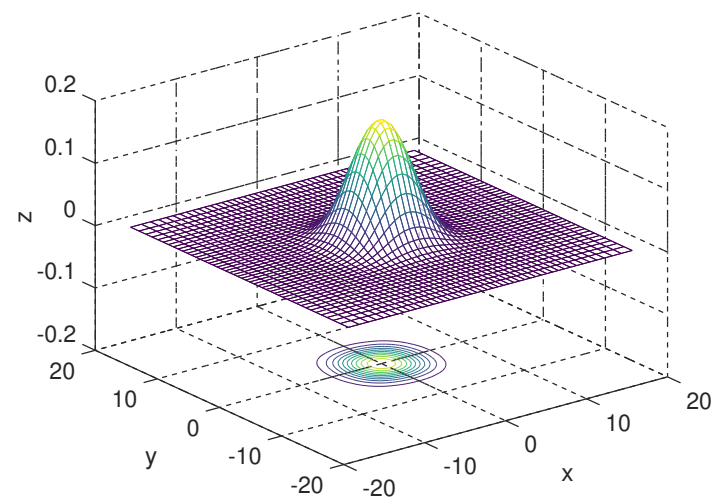

(a) $\bar{\Psi}_{00}(x, y), \quad E_{00}=-0.8002386220129$

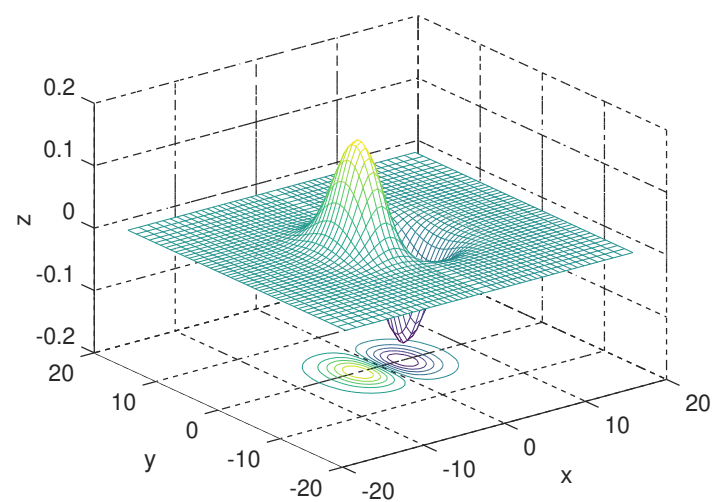

(c) $\bar{\Psi}_{01}(x, y), \quad E_{01}=-0.5544027490889$

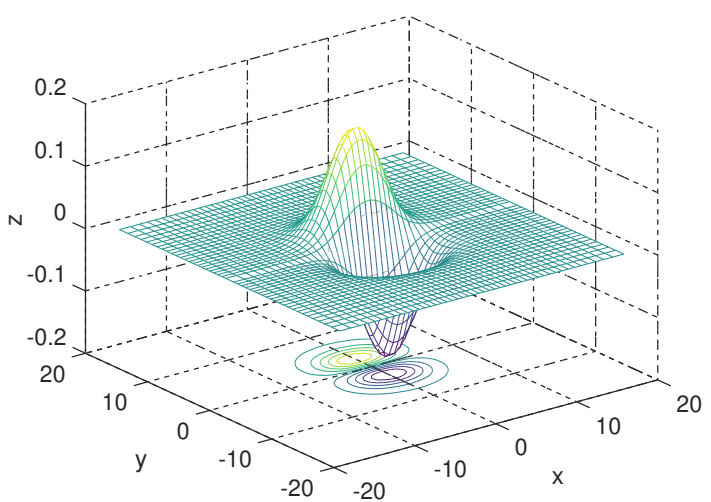

(b) $\bar{\Psi}_{10}(x, y), \quad E_{10}=-0.5544027490889$

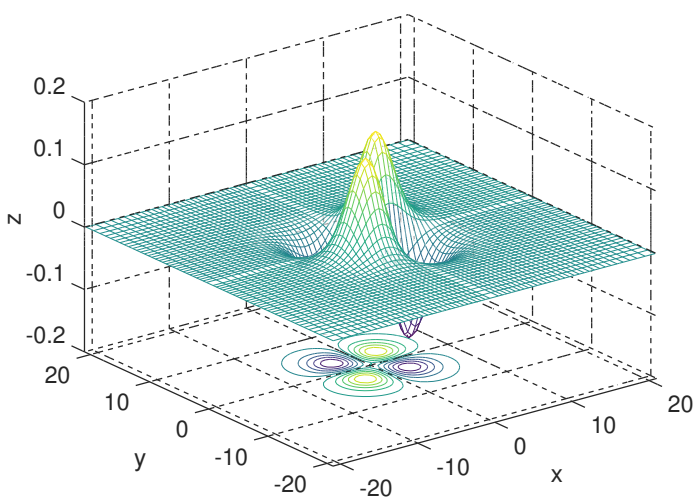

(d) $\bar{\Psi}_{11}(x, y), \quad E_{11}=-0.3214069787951$

Figure 2. First four normalized wavefunctions of the Gaussian type potential in (3.2) when $\beta=0.001$.

Table 3. Comparison of the truncation sizes of the LPM and HPM for the discrete states of the Gaussian potential in (3.2) when $\beta=0.001$.

\begin{tabular}{llll}
\hline$N_{L P M}$ & $N_{H P M}$ & $\alpha_{\text {opt }}$ & $E_{2 m, 2 n}$ \\
\hline 30 & 60 & 0.5 & -0.8002386220129 \\
& & 0.3 & -0.2706276650 \\
& & -0.2525793455 \\
\hline \multirow{3}{*}{30} & & & $E_{2 m, 2 n+1}$ and $E_{2 m+1,2 n}$ \\
\cline { 2 - 2 } & & 0.5 & -0.554402749088 \\
& & 0.3 & -0.064141040 \\
\hline \multirow{3}{*}{60} & 0.2 & -0.00459 \\
\hline
\end{tabular}

which has a finite number of discrete states between $-1<E_{m, n}<0$ together with a continuous spectrum over the entire positive real axis for small values of the real parameter $\beta$.

Next, as a third example, we consider the hyperbolic secant potential

$$
V(x, y)=-m(m+1) \operatorname{sech}^{2}\left[\left(x^{4}+y^{4}\right)^{1 / 2}\right]
$$


Table 4. Improved results for the discrete states of the Gaussian potential in (3.2) when $\beta=0.001$ by using LPM.

\begin{tabular}{lll}
\hline$N_{L P M}$ & $\alpha_{\text {opt }}$ & $E_{2 m, 2 n}$ \\
\hline 42 & 0.5 & -0.8002386220129 \\
& 0.3 & -0.2706276648250 \\
& & -0.2525793454554 \\
\hline 35 & 0.5 & $-E_{2 m, 2 n+1}$ and $E_{2 m+1,2 n}$ \\
48 & 0.3 & -0.5544027490889 \\
60 & 0.2 & -0.06414104349 \\
\hline 48 & 0.5 & $E_{2 m+1,2 n+1}$ \\
\hline
\end{tabular}

where $m>0$. Like the Gaussian potential, there exist finitely many discrete states located on the negative real axis between $-m(m+1)<E<0$ together with the continuous spectrum on the whole positive real axis. Tables 3 and 5 presents the computed eigenvalues of the Gaussian type and hyperbolic secant potential by using the LPM and HPM.

Table 5. Comparison of the truncation sizes of the LPM and HPM for the discrete states of the hyperbolic secant potential in (3.3) when $m=5$.

\begin{tabular}{llll}
\hline$N_{L P M}$ & $N_{H P M}$ & $\alpha_{\text {opt }}$ & $E_{2 m, 2 n}$ \\
\hline 30 & 60 & 2.5 & -23.904156425734 \\
& & -8.2769165345 \\
& & -7.6075591202 \\
\hline \multirow{2}{*}{30} & & \multicolumn{2}{c}{$E_{2 m, 2 n+1}$ and $E_{2 m+1,2 n}$} \\
& & & -16.50509970087 \\
& & 2.5 & -2.343009 \\
\hline \multirow{2}{*}{60} & 1.5 & -0.53294 \\
\hline
\end{tabular}

For the last two potentials again we see that $N_{H P M} \approx 2 N_{L P M}=60$. Therefore, by increasing the truncation size $N_{L P M}$ of the present algorithm it is possible to obtain more accurate results than the HPM which are presented in Tables 4 and 6 . However, a remarkable slowing down of the convergence is met for the discrete states at the border of the continuum which is a common drawback of almost all methods even in the onedimensional case. This is because of the nonexistence of the contributions coming from the continuous spectrum eigenfunctions in the basis functions [3,11].

Finally, we take into account the quartic anharmonic oscillator

$$
V(x, y)=x^{2}+y^{2}+c_{4}\left(x^{4}+2 a x^{2} y^{2}+y^{4}\right)
$$

with $c_{4}>0$, and $-1 \leq a \leq 1$. Table 7 present the states $E_{2 m, 2 n}, E_{2 m, 2 n+1}, E_{2 m+1,2 n}$ and $E_{2 m+1,2 n+1}$ when $c_{4}=1000$ and $a=1$. For comparison we also tabulate the results from [13] that employs the certain trigonometric basis set in a Rayleigh-Ritz variational scheme. We have executed our computer program in gfortran-4.8 by using quadruple precision arithmetic so that the comparison of the numerical results with those of [13] becomes more meaningful and informative. Clearly, our results are slightly better than those of [13] where 17-18 decimal points of accuracy was obtained with the truncation size of $N=22$. On the other hand, with the same truncation size, we obtain $18-22$ decimal points of accuracy. Moreover, the algorithm of [1] is also implemented in Fortran programming language and 
Table 6. Improved results for the discrete states of the hyperbolic secant potential in (3.3) when $m=5$ by using LPM.

\begin{tabular}{lll}
\hline$N_{L P M}$ & $\alpha_{\text {opt }}$ & $E_{2 m, 2 n}$ \\
\hline 30 & 2.5 & -23.904156425734 \\
33 & 2.0 & -8.27691653456 \\
& & -7.60755912019 \\
\hline 30 & 2.5 & $E_{2 m, 2 n+1}$ and $E_{2 m+1,2 n}$ \\
60 & 1.5 & -16.5050997008722 \\
& & -2.34300909747 \\
60 & 2.0 & -0.53296325 \\
\hline
\end{tabular}

executed in quadruple precision arithmetic. Again, the doubling $N_{H P M} \approx 2 N_{L P M}=44$ in the truncation sizes of the HPM and LPM is clear. Note that the HPM produces the full spectrum at once, however, while reporting in Table 7 we split it into four states to compare the results easily.

Table 7. First few energy eigenvalues of the quartic anharmonic oscillator in (3.4) when $c_{4}=1000, a=1$.

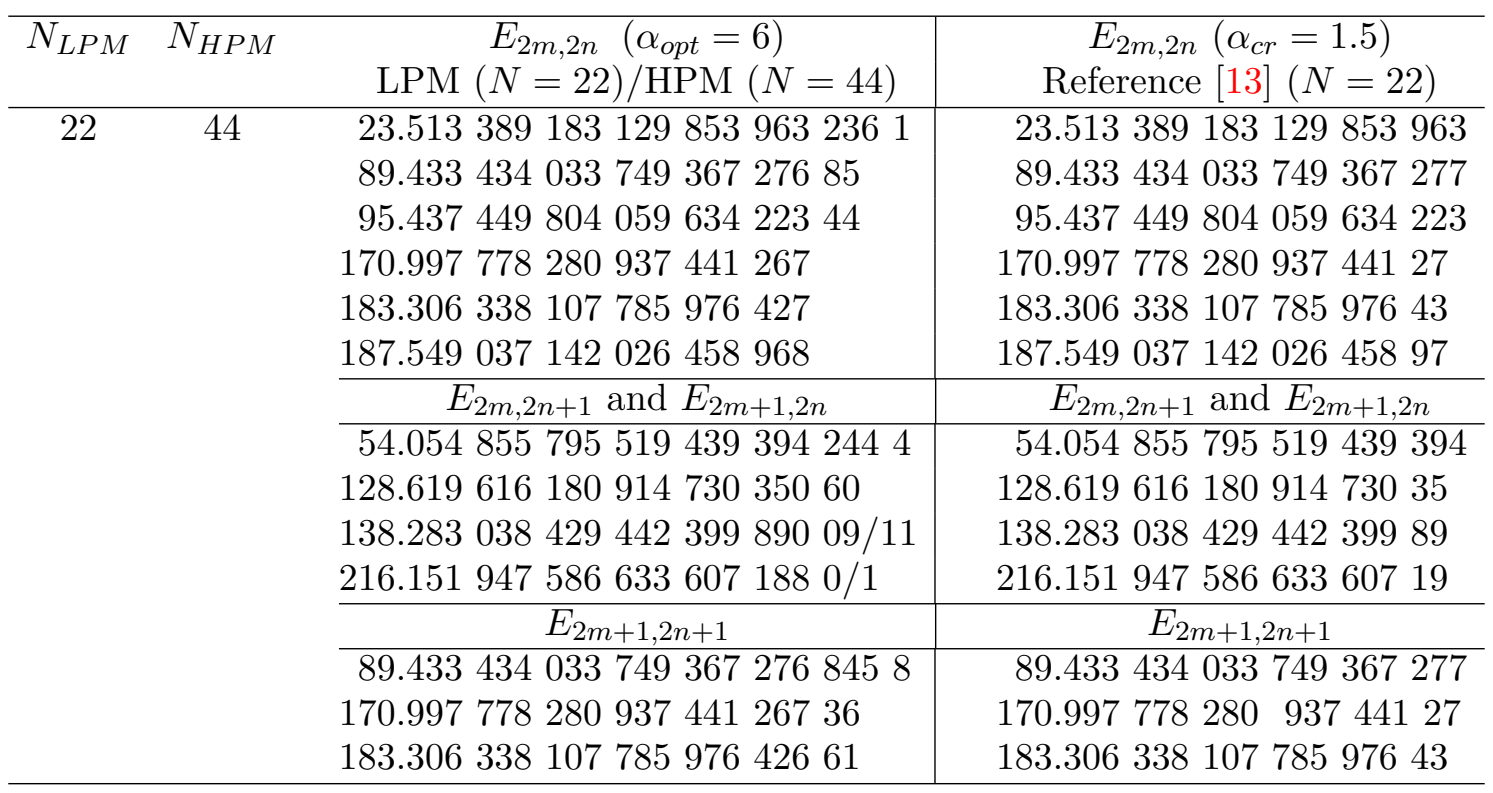

Closeness in the numerical results with the same truncation size is not too much surprising since both the trigonometric and Laguerre basis sets are somehow the exact solutions of the problem in (1.1). More specifically, the former is the exact solution of (1.1) over a finite rectangle $(x, y) \in(\alpha, \alpha) \times(\beta, \beta)$ with Dirichlet boundary conditions in which the potential is in the form of a rectangular box with impenetrable walls [13]. The latter is the solution of the system (1.1)-(1.2) under the two-dimensional harmonic oscillator potential $V(x, y)=x^{2}+y^{2}$ (see the transformed equation (1.12) together with (1.13)-(1.14) when $\alpha=1)$. 


\section{Conclusion}

In this article, the two-dimensional Schrödinger equation over the whole real plane with symmetric nonseparable potentials is solved numerically by using the LPM. Transformation of the problem over the first quadrant enabled us to treat the states $E_{2 m, 2 n}, E_{2 m, 2 n+1}$, $E_{2 m+1,2 n}$ and $E_{2 m+1,2 n+1}$ separately. By this way, instead of diagonalizing a matrix of size $4 N^{2} \times 4 N^{2}$ we compute the eigenvalues of four matrices of size $N^{2} \times N^{2}$ to obtain the same number of eigenvalues to a certain accuracy.

It is known that the numerical eigenvalue problems suffer from the problem of computing the full set of eigenvalues with a uniform accuracy [14]. Only the portion of the eigenvalues can be obtained with a desired accuracy for a fixed truncation order $N$. Therefore, the treatment of the states $E_{2 m, 2 n}, E_{2 m, 2 n+1}, E_{2 m+1,2 n}$ and $E_{2 m+1,2 n+1}$ by separate basis sets quadruples the number of high accurate eigenvalues for the same fixed truncation size $N$.

On the other hand, comparison of the present numerical results with those obtained by applying the HPM [1] reveals evidently that $N_{H P M} \approx 2 N_{L P M}$. Clearly, the use of LPM instead of HPM halves the number of points and reduces the cost by a factor of two in each direction which strongly supports the main argument of the present study. Note that, we do not claim that the LPM is superior to the HPM from the efficiency or accuracy point of view in general. In fact, even the direct comparison of these two methods is not meaningful since the Hermite and Laguerre polynomials are defined over the whole real line and half line, respectively. However, here we have shown that if the system (1.1)(1.2) has the reflection symmetries, that is, both the differential equation in (1.1) and the boundary conditions in (1.2) are invariant under the replacement of the independent variables $x$ and/or $y$ by their negatives, then the transformation of the problem over the first quadrant and the use of LPM with $\gamma= \pm \frac{1}{2}$ separate the states $E_{2 m, 2 n}, E_{2 m, 2 n+1}$, $E_{2 m+1,2 n}$ and $E_{2 m+1,2 n+1}$, and hence, halve the truncation size $N$ in each direction. This reduction is important since in two dimension the size of the resulting discrete system increases as the square of the truncation size. Alternatively, without transforming the equation, one may use the even or odd indexed Hermite polynomials as basis sets to separate the above four states. Actually, both approaches can be regarded as equivalent if we remember the interrelations

$$
H_{2 n}(x)=(-1)^{n} 2^{2 n} n ! L_{n}^{-1 / 2}\left(x^{2}\right)
$$

and

$$
H_{2 n+1}(x)=(-1)^{n} 2^{2 n+1} n ! x L_{n}^{1 / 2}\left(x^{2}\right)
$$

between the Hermite and Laguerre polynomials. Unfortunately, direct use of $H_{2 n}(\xi)$ or $H_{2 n+1}(\xi)$ in (2.2) does not allow the separation of the above states. Thus, in this study we have presented the use of $L_{n}^{\gamma}(\xi)$ with $\gamma= \pm \frac{1}{2}$ after the transformation of the problem over the first quadrant in order to reduce the total cost by a factor of four. As a result, the same accuracy of the HPM for the numerical eigenvalues is obtained with considerably low cost which is in accordance with the main aim of this study. Remember that our claim was to present a pseudospectral method for the numerical solution of the two dimensional Schrödinger equation with symmetric nonseparable potentials which is more efficient than the HPM from a computational point of view.

\section{References}

[1] H. Alıc1, The Hermite pseudospectral method for the two-dimensional Schrödinger equation with nonseparable potentials, Comput. Math. Appl. 69 (6), 466-477, 2015.

[2] H. Alıcı and H. Taşeli, Pseudospectral methods for an equation of hypergeometric type with a perturbation, J. Comput. Appl. Math. 234, 1140-1152, 2010.

[3] M. Demiralp, N.A. Baykara, and H. Taşeli, A basis set comparison in a variational scheme for Yukawa potential, J. Math. Chem. 11, 311-323, 1992. 
[4] G.H. Golub and J.H. Welsch, Calculation of Gauss quadrature rules, Math. Comput. 23, 221-230 s1-s10, 1969.

[5] B.-Y. Guo, L.-L. Wang, and Z.-Q. Wang, Generalized Laguerre interpolation and pseudospectral method for unbounded domains, SIAM J. Numer. Anal. 43, 2567-2589, 2006.

[6] F.B. Hildebrand, Method of Applied Mathematics, McGraw-Hill, NewYork, 1956, pp. 319-323.

[7] G. Mastroianni and D. Occorsio, Lagrange interpolation at Laguerre zeros in some weighted uniform spaces, Acta Math. Hungar. 91 (1-2), 27-52, 2001.

[8] J. Shen, Stable and efficient spectral methods in unbounded domains using Laguerre functions, SIAM J. Numer. Anal. 38 (4), 1113-1133, 2000.

[9] J. Shen and L.-L. Wang, Some recent advances on spectral methods for unbounded domains, Commun. Comput. Phys. 5, 195-241, 2009.

[10] T. Tang, The Hermite spectral method for Gaussian-type functions, SIAM J. Sci. Comput. 14 (3), 594-606, 1993.

[11] H. Taşeli, Modified Laguerre basis for hydrogen-like systems, Int. J. Quantum Chem. 63, 949-959, 1997.

[12] H. Taşeli and H. Alıcı, The Laguerre pseudospectral method for the reflection symmetric Hamiltonians on the real line, J. Math. Chem. 41, 407-416, 2007.

[13] H. Taşeli and R. Eid, Eigenvalues of the two-dimensional Schrödinger equation with nonseparable potentials, Int. J. Quantum Chem. 59, 183-201, 1996.

[14] J.A.C. Weideman and L.N. Trefethen, Eigenvalues of second-order spectral differentiation matrices, SIAM J. Numer. Anal. 25, 1279-1298, 1988. 